\title{
EL REGIONALISMO SUDAMERICANO FRENTE A LA INTEGRACIÓN EUROPEA: UNA PERSPECTIVA DE ANÁLISIS DESDE CATEGORÍAS DIFERENTES
}

\author{
Michel Levi ${ }^{1}$
}

\begin{abstract}
El artículo se refiere al proceso de comparación y relación que existe entre el regionalismo suramericano, frente a la categoría de análisis de la integración europea. En general, los bloques de integración regional han sido confrontados a los fundamentos teóricos establecidos sobre la base de la integración europea. En la actualidad el objetivo de realizar la comparación ya no es encontrar la similitud con la integración europea, sino diferenciar bajo diversos criterios la dinámica de los procesos del regionalismo suramericano. Para el efecto, el trabajo utiliza el método de análisis cualitativo, a través de la revisión de literatura especializada producida por académicos en diferentes idiomas.
\end{abstract}

Palabras clave: regionalismo; Unión Europea; Suramérica; integración regional; modelos; indicadores.

\section{O REGIONALISMO SUL-AMERICANO E A INTEGRAÇÃO EUROPEIA: EXPLORAÇÃO DE DIFERENTES CATEGORIAS DE ANÁLISE}

\begin{abstract}
0 artigo se refere ao processo de comparação e relacionamento que existe entre o regionalismo sul-americano, em oposição à categoria de análise da integração europeia. Em geral, os blocos de integração regional foram confrontados com os fundamentos teóricos estabelecidos com base na integração europeia. Atualmente, o objetivo de fazer a comparação não é mais encontrar a semelhança com a integração europeia, mas distinguir sob diversas condições a dinâmica dos processos do regionalismo sul-americano. Para isso, o trabalho utiliza o método de análise qualitativa, por meio da revisão de literatura especializada produzida por acadêmicos em diferentes idiomas.
\end{abstract}

Palavras-chave: regionalismo; União Europeia; América do Sul; integração regional; modelos; indicadores.

\section{SOUTH AMERICAN REGIONALISM AND THE EUROPEAN INTEGRATION: EXPLORING DIFFERENT CATEGORIES OF ANALYSIS}

The article refers to the process of comparison and relationship that exists between South American regionalism, as opposed to the category of European integration analysis. In general, the regional integration blocs have been confronted with the theoretical foundations established based on European integration. Currently, the purpose of making the comparison is no longer to find the similarity with European integration, but to distinguish under many conditions the dynamics of

1. Dr. (c) en administración pública (ciencia política y derecho) en el Instituto de Estudios Políticos de Grenoble de la Universidad Grenoble Alpes (Francia); coordinador del Centro Andino de Estudios Internacionales de la Universidad Andina Simón Bolívar (sede Ecuador); e investigador del Centro de Estudios e Investigación sobre la Diplomacia, la Administración Pública y la Política (CERDAP2) (EA 7443), Sciences Po Grenoble, en la Universidad de Grenoble Alpes. Correo electrónico: <michel.levi@uasb.edu.ec>. Orcid: <https://orcid.org/0000-0002-4976-9270>. 
the processes of South American regionalism. To this end, the paper uses the method of qualitative analysis, through the review of specialized literature produced by academics in different languages.

Keywords: regionalism; European Union; South America; regional integration; models; indicators. JEL: F53.

DOl: http://dx.doi.org/10.38116/rtm23art1

Data de envio do artigo: 28/8/2020; Data de aceite: 17/9/2020.

\section{INTRODUCCIÓN}

El artículo presenta elementos que permiten comprender por qué la categoría regionalismo suramericano ha sido analizada a partir de indicadores establecidos, desde la perspectiva teórica y práctica de la integración europea, a través de programas de investigación como el regionalismo comparado.

Estos indicadores se utilizan para establecer vínculos en la construcción de los diferentes procesos de integración regional a nivel global. La literatura especializada se enfoca en plantear al proceso europeo como un modelo de integración, cuyos avances y desarrollo conceptual generan los parámetros utilizados generalmente por actores académicos para confrontar diferentes esquemas regionales o de integración regional (Levi, 2011).

El trabajo se fundamenta en la investigación cualitativa, sustentada en la revisión de literatura especializada, producida por investigadores europeos y suramericanos que trabajan diferentes lógicas de construcción de las estructuras a nivel de las categorías de análisis, establecidas como integración europea y regionalismo suramericano.

La literatura especializada, reflejada en autores de diferentes tendencias y nacionalidades como José Briceño-Ruiz, Cintia Quiliconi, Raúl Salgado Espinoza, Kevin Parthenay, Tobias Lenz, Olivier Dabène o Andrés Malamud, realiza aproximaciones desde diferentes disciplinas del conocimiento, generalmente la ciencia política, a través de las teorías de la integración regional, de la economía política internacional, la sociología política o el derecho comunitario.

La literatura que analiza y compara los procesos regionales suramericanos ha buscado encontrar las coincidencias posibles con el referente europeo, sobre todo en la construcción institucional supranacional, considerada como "uno de los rasgos más destacados del proceso de integración” (Briceño-Ruiz, 2018, p. 58).

Por otra parte, se ha puesto sobre la mesa de discusión el tema de la integración europea como modelo, un comparator, es decir "el caso que permite evaluar otros casos de integración en el mundo" (Briceño-Ruiz, 2018, p. 24). En este contexto, desde Europa, Tobias Lenz (Lenz y Nicolaidis, 2019, p. 78), por ejemplo, plantea que es necesario "un análisis crítico de la idea, discurso y 
práctica de Europa como un modelo, sin dejar de lado el valor agregado y aporte de la construcción europea para el resto del mundo".

De acuerdo a Parthenay (2019, p. 101), desde la perspectiva de la integración europea se han realizado una serie de investigaciones para establecer la existencia de una difusión normativa y/o del "modelo" institucional europeo, fundamentadas en el interés de los actores y la forma en que los procesos regionales lo duplican.

La diferencia de aproximación de los investigadores nos permite comprender desde las categorías conceptuales que, a través de la explicación de la integración europea como un posible "modelo" de comparación, o de difusión de un poder institucional o normativo, o de confrontación teórica, puede determinarse que el regionalismo suramericano corresponde a lógicas de implementación relacionadas, diferentes o contrarias a una perspectiva eurocentrista de la integración.

\section{LA LÓGICA DEL REGIONALISMO SURAMERICANO ACTUAL FRENTE A LA CATEGORÍA INTEGRACIÓN EUROPEA}

Durante los últimos veinticinco ańos el regionalismo suramericano ha tenido una importante dinámica que ha llevado a la creación de una serie de procesos de integración regional y procesos regionales, tales como la Comunidad Andina (CAN), Mercado Común del Sur (Mercosur), Unión de Naciones Suramericanas (Unasur), Alianza del Pacífico (AP), Alianza Bolivariana para los pueblos de nuestra América (Alba).

Académicos latinoamericanos han realizado análisis, durante buena parte de esta década, de las condiciones que han generado esta diversidad de bloques regionales. Sus investigaciones han determinado corrientes de pensamiento expresadas a través de series de publicaciones en diferentes idiomas y contextos, tales como el regionalismo post liberal (Da Motta y Ríos, 2007; Sanahuja, 2010, 2012, 2016), el regionalismo post hegemónico (Riggirozzi Y Tussie, 2012), el regionalismo estratégico, social y productivo (Briceño-Ruiz, 2011), entre los más determinantes.

Desde nuestro punto de vista, la perspectiva del regionalismo "a la carta" (Quiliconi y Espinoza, 2017) explica con bastante precisión los cambios operados en la región a partir de los años noventa del siglo XX, dominada por la ideología neo liberal del Consenso de Washington, que determina la corriente del regionalismo abierto, que se transforma en la primera década del siglo XXI en regionalismo post liberal (agenda de Brasil) o post hegemónico (agenda de Venezuela) con mecanismos regionales que no se limitan a los aspectos económicos e incluyen agendas políticas y sociales (Quiliconi y Espinoza, 2017, p. 22-23). 
Las agendas de los procesos del regionalismo suramericano, se configuran bajo diversos andamiajes institucionales, que se implementan, en principio, a través de políticas regionales, creación normativa y mecanismos de toma de decisiones (Lenz y Nicolaidis, 2019) como indicadores que pueden definir el alcance del regionalismo en cada uno de los mecanismos mencionados.

El alcance del regionalismo es diferente si nos referimos a los procesos de integración del regionalismo abierto o a los procesos regionales, que son considerados como parte de las corrientes del regionalismo contemporáneo del siglo XXI (Quiliconi y Espinoza, 2017).

En el primer caso, desde la perspectiva de la narrativa convencional, los mecanismos funcionales que influenciaron en la creación de la CAN y Mercosur se establecieron durante la última década del siglo XX, bajo la cooperación de la integración europea (Malamud, 2020, p. 4).

En el regionalismo abierto, el análisis de los procesos de integración regional suramericanos ha sido confrontado a partir de las teorías que explican el desarrollo de la integración europea. La relación que se establece entre sus estructuras puede comprenderse a partir de una serie de mecanismos como las relaciones interregionales, la difusión del poder normativo o la transferencia a través de la cooperación (Malamud, 2020; Parthenay, 2019; Briceño-Ruiz, 2018; Lenz y Nicolaidis, 2019).

Los mecanismos del regionalismo contemporáneo, se establecieron a partir de la primera década del siglo XXI, cuando se crearon la Unasur, Alba y AP, sobre una base funcional diversa, que responde a intereses políticos de los países que los conforman y a la agenda que buscan aplicar de acuerdo a su especialización (Quiliconi y Espinoza, 2017, p. 29, 35).

En el caso de los procesos del regionalismo contemporáneo, su estructura no corresponde a una lógica convencional de integración regional, por lo que surge el cuestionamiento sobre la posibilidad de ser analizados como expresiones comparables de regionalismo (Quiliconi y Espinoza, 2017, p. 35).

Sin embargo, en estos casos podrían confrontarse una serie de indicadores específicos establecidos para procesos regionales, no necesariamente de integración regional, que parten del análisis de los objetivos y mecanismos que se han configurado en sus documentos o tratados fundacionales.

En efecto, el análisis de los procesos del regionalismo abierto se lo realiza desde la lógica de la integración regional, específicamente se aplican las teorías que han sustentado el desarrollo del proceso europeo, que es utilizado como modelo o referente en el marco de programas de investigación como el regionalismo comparado (Briceño-Ruiz, 2018, p. 9-15; Malamud, 2020, p. 4-9). 
Sin embargo, en los procesos del regionalismo contemporáneo la comparación es más estructurada en torno a una serie de indicadores que permiten comprender la naturaleza de los mecanismos, toda vez que no todos pueden considerarse de integración, en función de la especialización para la que han sido establecidos (Quiliconi y Espinoza, 2017, p. 17-22).

En este contexto resulta útil la categorización que realiza Briceño-Ruiz (2018, p. 12-14) para establecer la diferencia entre el análisis de la integración regional, tal como la plantean los clásicos (Bela Balassa, Haas) bajo diferentes categorías como integración regional, integración económica, integración política y cooperación, frente al análisis del regionalismo, categoría que se utiliza como referencia, sobre todo a partir de finales del siglo XX y comienzos del siglo XXI (Hettne, Soderbaum) que "no se limita a la integración" e incluye "formas de acción conjunta como la cooperación y la concertación” (Briceño-Ruiz, 2018, p. 14).

En esta relación cabe destacar los diferentes análisis y aproximaciones que se hacen en torno a la lógica del regionalismo suramericano frente al contexto teórico de la categoría integración regional europea.

\section{LAS CATEGORÍAS DE ANÁLISIS DE LA INTEGRACIÓN REGIONAL EUROPEA FRENTE A LA LÓGICA DEL REGIONALISMO SURAMERICANO}

La aproximación que se realiza para explicar la fenomenología y alcances que tienen (o deberían tener) los mecanismos regionales suramericanos, en nuestro caso específico de estudio, se ha establecido a través de la teoría de la integración regional, fundamentada desde la perspectiva europea.

Algunos investigadores que trabajan la relación de la teoría con diferentes casos de integración, han considerado estas posiciones como eurocéntricas, sin que signifique que existe colonización de las ideas o de los métodos de análisis (Lenz y Nicolaidis, 2019, p. 88-90). En algunos casos refleja un desconocimiento de la producción científica que explica los mecanismos regionales desde un contexto suramericano o latinoamericano (Briceño-Ruiz, 2018, p. 11) y en las motivaciones que responden a las circunstancias y necesidades propias para la creación de proyectos regionales (Briceño-Ruiz, 2018, p. 34).

Por otra parte, es necesario comprender que las teorías de la integración deben partir de la necesidad de universalizar el conocimiento, producido a través del análisis y confrontación de casos, que pueden ser analizados bajo sistemas de indicadores utilizados para explicar diferentes categorías que interactúan en los procesos a nivel global (Levi, 2011, p. 238-240).

La diferencia de aproximación entre el análisis de la integración regional y el regionalismo es importante para establecer el alcance en la explicación de 
los diferentes mecanismos regionales suramericanos. Por ejemplo, desde la perspectiva de la integración considerada a partir de la experiencia europea, que corresponde a la definición establecida por Haas y complementada por Schmitter (Malamud, 2013, p. 2-3), aplicada a los procesos de integración regional como CAN y Mercosur, puede determinarse que se trata de casos de "no integración" (Malamud, 2020, p. 9), desde un análisis que confronta el cumplimiento conceptual de la integración regional frente a la evolución de los objetivos de esos bloques.

En el caso anterior, la determinación de "no integración" podría considerarse como un caso de regionalismo, sin embargo, es necesario considerar que la aplicación adecuada de la contextualización en el análisis de lo que es integración, permite considerar con prudencia el alcance de la definición anterior (Briceño-Ruiz, 2018, p. 29-32, 37).

Es decir, se requiere mayores elementos de contexto y motivación para comprender la lógica de esos bloques de integración y sus resultados en el momento actual, que se sujetan a dinámicas e intereses diferentes a los explicados desde el contexto teórico fundamentado en la experiencia europea.

El regionalismo, por su parte, permite comprender con mayor amplitud el ámbito de existencia de bloques regionales que no se limitan a la experiencia europea y dimensionan el proceso de globalización y los cambios en el sistema político que operan a partir de finales del siglo XX (Briceño-Ruiz, 2018, p. 22). Esta categoría explica con mayor precisión la existencia de procesos regionales especializados como la AP, Alba o Unasur, que no coinciden necesariamente con los parámetros de la reflexión teórica de la integración regional.

De forma especial durante el periodo del regionalismo abierto se buscó vincular la creación de los bloques de integración suramericanos, como la CAN y el Mercosur, al desarrollo funcional y normativo de la integración europea. La historia en el momento actual opera un cambio en la lógica del análisis, en razón de que los investigadores buscan explicaciones para establecer las diferencias que existen en la dinámica de la integración regional y del regionalismo suramericano (Malamud, 2020, p. 9).

Las diferencias que se producen entre la dinámica de la integración europea, fundamentada en la serie de teorías que explican sus avances, en los últimos veinticinco ańos aproximadamente, coinciden con la puesta en marcha de los cambios introducidos en los Tratados de Maastricht (1992), Ámsterdam (1997) y particularmente Lisboa (2007).

Estos cambios dan como resultado en una primera fase el fortalecimiento de los vínculos y mecanismos inter regionales entre la Unión Europea (UE) y los 
bloques suramericanos y el centroamericano, que se reflejan en eventos como la cumbre UE-ALC celebrada en Río de Janeiro en 1999. En la cumbre participaron los jefes de Estado y de obierno de los países en las dos regiones, incluido el Caribe, que validaron al bloque europeo como una estructura de gobernanza regional en un contexto global.

El intercambio de experiencias y cooperación producido a través de los mecanismos inter regionales, permitió a los bloques de integración regional crear vínculos por una serie de actores que comprende no solo los Estados, sino agencias regionales e incluso la sociedad civil. Estas relaciones son generalmente asimétricas y han permitido a la estructura europea fortalecer a otros bloques de integración regional, con intercambio de experiencias y de cooperación (Malamud, 2020, p. 9-10).

El inter regionalismo, es decir la relación pura entre bloques, genera un intercambio de estándares de integración regional que pueden ser aprovechados por las partes en una relación asimétrica (Parthenay, 2019). Esta lógica considera a la integración europea como un poder normativo, que tiene capacidad de difundir alrededor del mundo su modelo institucional y normativo, así como considerarse un actor global con una estrategia para exportar su modelo de gobernanza regional (Parthenay, 2019).

Desde la aproximación de Parthenay (2019), la integración europea ha puesto a funcionar una estructura exportadora a través de un modelo de referencia para la integración regional. Sin embargo, en lo referente a los mecanismos de difusión de un modelo de integración, considera que la literatura académica producida, sobre todo, por Anja Jetschke y Tobias Lenz aborda el análisis que cuestiona el hecho de que la integración europea pueda ser considerado como un modelo (Lenz y Nicolaidis, 2019).

Especialmente Lenz ha mantenido esta línea de investigación sobre la difusión del "modelo" de integración europea, hasta llegar a establecer una pregunta de base para comprender si realmente existe. ¿Qué implica un modelo? Sin negar el valor agregado que puede tener la lógica de la integración europea, hasta qué punto puede ser confrontada como un modelo de gobernanza regional (Lenz y Nicolaidis, 2019, p. 80-84).

Las acepciones de modelo pueden tener matices diferenciadores. Por una parte, un modelo puede ser una representación de algo que permite su reproducción. Por otra parte, el modelo podría significar una aspiración "a ser como algo", que puede ser imitado. Finalmente, una acepción diferente sería considerar al modelo como algo que sirve como inspiración y es objeto de reinterpretación (Lenz y Nicolaidis, 2019, p. 80). 
El modelo se convierte en un tipo ideal, una versión simplificada, pura, determinada de la realidad, para representar también un esquema de perfección, que puede ser imitada, como un rol de modelo. En el medio artístico, por ejemplo, el o la modelo que posa implica que puede ser sujeto de una interpretación que refleja o no la realidad.

En este sentido el modelo de la integración europea, representa y depende de quién lo considera como tal, de forma que esta representación que se hace de la integración europea puede referirse a una serie de elementos que la constituyen como en el caso de políticas, instituciones o mecanismos de toma de decisiones. Sus valores y principios constitutivos también forman parte de esa visión de modelo, que puede aplicarse de forma más amplia como parámetros de libertad y paz, por el lado positivo, o de limitada soberanía.

Sin embargo, por la variedad de acercamientos a lo que puede ser el modelo europeo, este no existe como lo conocemos, sino que representa una serie de parámetros que los actores consideran que son indispensables para su correcto funcionamiento, como las normas (UE), instituciones y sus prácticas. Esta lógica de interpretación positiva y favorable a la dinámica de la construcción europea que se fundamenta en la visión de los aspectos positivos, que en muchos casos proyecta los deseos y perspectivas de quienes analizan, más que las realidades menos positivas que pueden existir en estos elementos o categorías de análisis (Lenz y Nicolaidis, 2019, p. 80-84).

Tanto Lenz como Parthenay se refieren al mecanismo de difusión del poder normativo europeo, como las diversas formas en que la integración europea influencia la gobernanza global, a través de sus normas o mecanismos institucionales, o la promoción de la democracia, de los derechos humanos y de las libertades. El poder normativo también implica la capacidad de modelar el concepto de lo que es normal, que convierte a la estructura europea en un referente fuera de sus fronteras (Parthenay, 2019, p. 100).

Las formas y mecanismos de difusión son amplias y corresponden a un análisis diferenciado del que se realiza en este artículo. Como referencia podemos señalar que los germanos parlantes han sido prolijos en la investigación sobre las causas, condiciones, mecanismos y estrategias de difusión, entre los que destacamos los trabajos realizados por Anja Jetschke, Tobias Lenz, Tania Börzel, Tomas Risse, Sabine Saurugger.

Un trabajo de referencia que resaltamos sobre la difusión es el de Börzel y Risse (2012), que se refiere a tres dimensiones de acción social: la razón instrumental, la razón normativa y la razón comunicacional, que a su vez permiten identificar cinco mecanismos para favorecer la difusión de ideas y normas (Parthenay, 2019, p. 99-100). 
La motivación de los actores y la forma como los mecanismos regionales duplican el modelo institucional europeo (Briceño-Ruiz, 2018, p. 34; Parthenay, 2019, p. 102), son necesarias para comprender el alcance de la difusión del poder normativo de la integración europea en los mecanismos regionales suramericanos, en nuestro caso de estudio.

El enfoque de este análisis toma en cuenta la teoría del isomorfismo institucional, para explicar la similitud de las estructuras institucionales creadas por los bloques de integración regional suramericanos sobre el referente teórico de la integración europea (Dabène, 2012, p. 88-101; Parthenay, 2019, p. 107, 112-117).

La construcción de una arquitectura institucional, del proceso de toma decisiones y de difusión normativa que pueden considerarse similares en la forma, difieren en su alcance, objetivos y mecanismo en los procesos de integración suramericanos (CAN, Mercosur) frente a la construcción, motivación y contexto de la integración europea. La dinámica del regionalismo suramericano, en sus diferentes mecanismos regionales y de integración regional, se diferencia en la teoría y práctica, del desarrollo de la integración europea en los últimos veinticinco años.

\section{CONCLUSIÓN}

En el artículo hemos utilizado una serie de categorías para comprender la relación e influencia existente entre el regionalismo suramericano y la integración europea.

Las categorías utilizadas son resultado de la revisión de literatura académica, para comprender por qué en el momento actual el análisis no se encuentra determinado a encontrar las coincidencias posibles entre el regionalismo suramericano y el referente europeo.

Durante el desarrollo de la investigación, se determinó en primer lugar una serie de investigadores con proyectos orientados a explicar el entramado de las relaciones que pueden darse entre bloques regionales diversos, sobre la base conceptual del regionalismo y la teoría de la integración regional.

La integración europea ha estado determinada como categoría de comparación, a partir del desarrollo de las teorías que explican las dinámicas de los bloques regionales en clave de integración regional. Desde esta perspectiva, los bloques regionales que pueden explicarse a partir de la categoría del regionalismo quedan limitados en la exposición que se lleva a cabo en esta investigación.

La diversidad de literatura académica nos llevó a concentrarnos en la revisión de los productos más recientes, que tienen referencias a otras publicaciones sobre 
la materia consideradas indispensables para comprender el contexto del trabajo y determinar el alcance del análisis.

Los autores que producen investigación y resultados en el tema, tienen una importante trayectoria académica, y llevan tiempo trabajando en el desarrollo de la temática analizada.

El tema es novedoso porque refleja la diversidad de aproximaciones que puede tener el análisis de la categoría integración europea como referente del regionalismo suramericano. La riqueza del debate radica en las categorías utilizadas para comprender las referencias existentes en los bloques regionales como las que enumeramos a continuación: teorías de integración regional, regionalismo, eurocentrismo, inter regionalismo, poder normativo, modelo, difusión e isomorfismo institucional.

Desde las categorías enunciadas, la relación existente en la construcción de los bloques regionales sobre la base teórica de la categoría integración europea es muy limitada por la diferencia en el desarrollo, motivación, alcance, contexto, dinámica, que permiten establecer una serie de indicadores útiles para realizar un análisis comparativo, para determinar las diferencias existentes en una aparente similitud.

Coincidimos con Fredrik Söderbaum (Briceño-Ruiz, 2018, p. 31) al señalar la importancia de no limitarse al estudio desde la perspectiva de especialización en una región, sino desde un análisis que no limita la comprensión y motiva al conocimiento universal:

la especialización regional tiende a llevar a los académicos a crear una caja de herramientas conceptuales y teorías que son desarrolladas desde y para su propia "región", sin realmente tratar de comprometerse en otros casos o discursos que le hacen competencia.

Tal provincianismo evita el desarrollo de un discurso más general y universal. Impide que los académicos reconozcan que ellos pueden estar analizando el mismo problema, pero con diferentes lenguajes y conceptualizaciones (Söderbaum, 2013, p. 4).

\section{REFERENCIAS}

BÖRZEL, T.; RISSE, T. From Europeanisation to diffusion: introduction. West European Politics, v. 35, n. 1, p. 1-19, 2012.

BRICEÑO-RUIZ, J. Del regionalismo estratégico al regionalismo social y productivo: las transformaciones del modelo de integración en el Mercosur. In: . (Ed.). El Mercosur y las complejidades de la integración regional. Buenos Aires: TESEO, 2011. p. 121-162.

Las teorías de la integración regional: más allá del eurocentrismo. Bogotá: Universidad Cooperativa de Colombia, 2018. 
DA MOTTA, P.; RÍOS, S. O regionalismo pós-liberal na América do Sul: origens, iniciativas e dilemas. Santiago: Cepal, 2007. (Série Comércio Internacional, n. 62).

DABÈNE, O. (2012) Explaining Latin America's fourth wave of regionalism: regional integration of a third kind. In: CONGRESS OF THE LATIN AMERICAN STUDIES ASSOCIATION, 2012, San Francisco. Proceedings... San Francisco: [s.n.], 2012.

LENZ, T.; NICOLAIDIS, K. EU-topia? A critique of the European Union as a model. Culture, Practice and Europeanization, v. 4, n. 2, p. 78-101, 2019.

LEVI, M. La Unión Europea y la nueva integración latinoamericana: parámetros de comparación aplicados en diferentes estudios sobre los procesos de integración. Comentario Internacional, n. 11, p. 217-251, 2011.

MALAMUD, A. Overlapping regionalism, no integration: conceptual issues and the Latin American experiences. Florence: European University Institute, 2013. (RSCAS Working Paper).

Mercosur and the European Union: comparative regionalism and interregionalism. Oxford Research Encyclopedia of Politics, p. 1-26, 2020.

PARTHENAY, K. Revisiter le pouvoir normatif européen: le double visage de l'UE en Amérique Centrale. Politique Européenne, v. 63, n. 1, p. 96-124, 2019. Disponible en: <https://doi.org/10.3917/poeu.063.0096>.

QUILICONI, C.; ESPINOZA, R. S. Latin American integration: regionalism à la carte in a multipolar world? Colombia Internacional, v. 92, p. 15-41, 2017. Disponible en: <dx.doi.org/10.7440/colombiaint92.2017.01>.

RIGGIROZZI, P.; TUSSIE, D. (Ed.). The rise of post-hegemonic regionalism: the case of Latin America. London: Springer, 2012.

SANAHUJA, J. A. La construcción de una región: Suramérica y el regionalismo posliberal. In: CIENFUEGOS, M.; SANAHUJA, J. A. (Coord.). Una región en construcción: Unasur y la integración de América del Sur. Barcelona: CIDOB, 2010. p. 87-134.

Regionalismo post-liberal y multilateralismo en Sudamérica: el caso de Unasur. In: SERBIN, A.; MARTÍNEZ, L.; RAMANZINI JÚNIOR, H. (Coord.). Anuario de la integración regional de América Latina y el Gran Caribe. Buenos Aires: Cries, 2012. p. 19-71.

- Regionalismo e integración en América Latina: de la fractura Atlántico-Pacífico a los retos de una globalización en crisis. Pensamiento Propio, v. 44, n. 21, p. 29-75, 2016. 
SÖDERBAUM, F. What's wrong with regional integration? The problem of Eurocentrism. Robert Schuman Centre for Advance Studies, n. 64, 2013.

\section{BIBLIOGRAFÍA COMPLEMENTARIA}

BRICEÑO-RUIZ, J. El ALBA: una discusión de su modelo, sus resultados y sus perspectivas. In: SERBIN, A.; MARTÍNEZ, L.; RAMANZINI JÚNIOR, $\mathrm{H}$. (Coord.). Anuario de la integración regional de América Latina y el Gran Caribe. Buenos Aires: Cries, 2014. p. 151-178.

DABÈNE, $O$. The politics of regional integration in Latin America: theoretical and comparative explorations. New York: Palgrave Macmillan, 2009.

. La quatrième vague de régionalisme. In: QUENAN, S.; CARLOS, V.;

SEBASTIEN, V. (Ed.). Les enjeux du développement en Amérique Latine: dynamiques socioéconomiques et politiques publiques. Deuxième édition. París: Institut des Amériques, 2014. p. 64-95.

HOFFMANN, A. M. Regional governance and policy-making in South America. New York: Palgrave Macmillan, 2019.

JETSCHKE, A.; LENZ, T. Does regionalism diffuse? A new research agenda for the study of regional organizations. Journal of European Public Policy, v. 20, n. 4, p. 626-637, 2013.

JETSCHKE, A.; MURRAY, P. Diffusing regional integration: the EU and Southeast Asia. West European Politics, v. 35, n. 1, p. 174-191, 2011.

LENZ, T. EU normative power and regionalism: ideational diffusion and its limits. Cooperation and Conflict, v. 48, n. 2, p. 211-228, 2013.

MALAMUD, A.; GARDINI, G. L. Has regionalism peaked? The Latin American quagmire and its lessons. The International Spectator: Italian Journal of International Affairs, v. 47, n. 1, p. 116-133, 2012.

RIGGIROZZI, P. Re-territorializando consensos: hacia un regionalismo post-hegemónico en América Latina. In: SERBIN, A.; MARTÍNEZ, L.; RAMANZINI JÚNIOR, H. (Coord.). Anuario de la integración regional de América Latina y el Gran Caribe. Buenos Aires: Cries, 2012. p. 129-151.

RIVAROLA-PUNTIGLIANO, A.; BRICEÑO-RUIZ, J. Resilience of regionalism in Latin America and the Caribbean. New York: Palgrave Macmillan, 2013. 